\title{
Editorial \\ Reivindicación de una identidad profesional
}

\author{
Juan Antonio RODRÍGUEZ-SÁNCHEZ ${ }^{(1)}$ \\ (1) Área de Historia de la Ciencia. Facultad de Medicina. Universidad de Salamanca \\ jarshm@usal.es
}

Recibido: 7-12-15

Aceptado: 9-12-15

El pasado también puede ser laboratorio en el que reacciones, variables y constantes inviten a la reflexión. Cuando los criterios de especialidad y especialización se redefinen, cuando las reglas de acceso al grupo profesional cambian, la historia permite contemplar las circunstancias actuales desde la singular perspectiva de las continuas transformaciones que posibilitaron la adaptación.

Dentro de seis meses se cumplirán doscientos años de la creación del Cuerpo de Médicos de Baños, según un Real Decreto de 29 de junio de 1816. Surgido a contracorriente, más por los propios intereses y filias de Fernando VII que por una demanda social o profesional ${ }^{1}$, el Cuerpo supuso el primer impulso para que apareciese en España un grupo de profesionales dedicados a tiempo parcial al conocimiento y mejor aplicación de las aguas minero-medicinales. Desde ese momento su existencia fue controvertida y objeto de ataques externos y enfrentamientos internos. El Cuerpo supuso una medicalización de las aguas con propiedades terapéuticas que generó una confrontación con los propietarios de las instalaciones balnearias y con aquellos usuarios que privilegiaban los aspectos lúdicos. Por su parte, los profesionales médicos se debatieron entre el deseo de pertenecer al mismo para gozar de sus prebendas y el airado rechazo a que se monopolizase el remedio hídrico. Desde dentro, el sistema de escalafón de antigüedad desincentivó a los recién ingresados y estimuló su ataque a los veteranos que disfrutaban de la posibilidad de elegir cada año los balnearios de mayor concurrencia y mejores instalaciones ${ }^{2}$.

Sin embargo, el Cuerpo fue también el núcleo desde el que se construyó una especialidad, se gestó un movimiento asociativo -la Sociedad Española de Hidrología Médica- que dio a luz una atribulada pero persistente publicación periódica (se haya llamado Anales o Boletín) $)^{3}$ y se pergeñó la completa institucionalización a través de una formación específica universitaria (desde 1912 con la Cátedra de Hidrología Médica) ${ }^{4-5}$ y de especialización clínica (legislativamente desde $1955 \mathrm{y}$, en la práctica, desde 1978 en la Escuela Profesional de Hidrología Médica) ${ }^{6}$. 
Con luces y, tal vez con mayor frecuencia, sombras, el Cuerpo sirvió para articular a un grupo de profesionales con un perfil científico y asistencial con características bien diferenciadas y una justa aspiración a su pleno reconocimiento. El termalismo social y los cambios de la industria balnearia posibilitaron para algunos finalmente esa dedicación a tiempo completo propia de la especialización, aunque ya el Cuerpo se hubiese extinguido. Sin embargo, la identidad profesional, aunque minoritaria, había quedado reafirmada.

Las efemérides pueden ser meros acontecimientos para descubrir placas conmemorativas, pero también suponen la excelente oportunidad de mostrar públicamente la existencia de una identidad profesional bicentenaria capaz de resistir las actuales veleidades administrativas.

\section{REFERENCIA NORMALIZADA}

Rodríguez-Sánchez JA. Editorial. Reivindicación de una identidad profesional. Bol Soc Esp Hidrol Med. 2016; 31(1): 9-10

\section{BIBLIOGRAFIA}

1. Alonso Álvarez L, Vilar Rodríguez M, Lindoso Tato E. El agua bienhechora. El turismo termal en España 1700-1936. A Coruña: Observatorio Nacional del Termalismo, 2010.

2. Rodríguez-Sánchez JA. Institucionalización de la Hidrología Médica en España. En Maraver Eyzaguirre F (ed.) Establecimientos balnearios: historia, literatura y medicina. Madrid: Servicio de Publicaciones de la Universidad Complutense de Madrid Balnea 1, 2006, pp. 25-40.

3. Maraver F, Corvillo I. Historia de la Sociedad Española de Hidrología Médica. Madrid: Servicio de Publicaciones de la Universidad Complutense de Madrid - Balnea 2, 2006.

4. Maraver F, Álvarez-Sala JL, Armijo F, Crego M, Cuenca, C, De Jorge J, RodríguezSánchez JA. Cien años de la Cátedra de Hidrología Médica. Madrid: Servicio de publicaciones de la Universidad Complutense de Madrid - Balnea 7, 2012.

5. Rodríguez-Sánchez JA. Cómo se crea una cátedra: Hipólito Rodríguez Pinilla y la Hidrología Médica española. Bol Soc Esp Hidrol Med, 2012; 27(1):8-13.

6. Maraver F, Aguilera L, Corvillo I, Morer C, Armijo F. L'enseignement de l'Hydrologie à l'Université Complutense de Madrid. Trente années de l'École Professionnelle d'Hydrologie Médicale et d'Hydrothérapie. Presse Therm Clim, 2007; 144:167-76. 